\title{
Management of ankle valgus in a skeletally immature patient by tension band plate hemiepiphysiodesis: A case report
}

\author{
Rameshwar Datt ${ }^{1}$, Gunjar Jain ${ }^{2}$, Hira Lal Nag ${ }^{3}$, Shubhankar Shekhar ${ }^{1}$ \\ From ${ }^{1}$ Senior Resident, ${ }^{3}$ Professor, Department of Orthopaedics, All India Institute of Medical Sciences, New Delhi, ${ }^{2}$ Assistant Professor, SGT Medical \\ College Hospital and Research Institute, Gurugram, Haryana, India
}

\begin{abstract}
Temporary hemiepiphysiodesis is the procedure of choice to correct ankle valgus deformity in a skeletally immature patient. However, the literature is inconclusive regarding the ideal choice of implant and the timing of the surgery. In the current case report, a 9-year-old girl with multiple hereditary exostoses and unilateral ankle valgus deformity underwent tension band plate (TBP) hemiepiphysiodesis, and gained a modest correction at a rate of $0.61 \%$ month. After 18 months of follow-up, there were no implant-related complications, and the functional outcome was also good. This case report shows a promising result of medial malleolar temporary hemiepiphysiodesis using a TBP for ankle valgus deformity in the pediatric population.
\end{abstract}

Key words: Ankle valgus, Temporary hemiepiphysiodesis, Tension band plate

A nkle valgus is a rare deformity among the pediatric population, however, it is observed in $2-50 \%$ of the children with hereditary multiple exostoses (HME) [1]. Osteochondromas are frequently present in the lateral aspect of the distal tibia and medial aspect of the distal fibula, and it has been hypothesized that these lesions may hamper lateral growth resulting in valgus deformity at the ankle [2]. Other explanations regarding the cause of the ankle valgus in HME patients are relative shortening of the fibula and tapering of the lateral aspect of distal tibial epiphysis [2]. Ankle valgus if left untreated, may lead to disturbed vocational training, limited recreational activities, and degenerative changes in the joint [1]. It can be managed by temporary hemiepiphysiodesis, using either a hemiepiphyseal stapling (HS), a transphyseal medial malleolar screw (MMS), or a tension band plate (TBP). However, a common consensus has not been reached regarding the ideal choice of implant. Furthermore, the determination of the optimum age for hemiepiphysiodesis, such that we can prevent repeated surgeries, is still not possible.

In the current case report, we have achieved a modest rate of correction (ROC) and good functional outcome with TBP hemiepiphysiodesis.

\begin{tabular}{|l|c|}
\hline \multicolumn{2}{|c|}{ Access this article online } \\
\hline Received - 23 September 2021 \\
Initial Review - 08 October 2021 \\
Accepted - 13 October 2021
\end{tabular}

\section{CASE REPORT}

A 9-year-old female child accompanied by her mother came to our outpatient department with complaints of multiple swellings over her lower limbs and a deformity on her left ankle, which she noticed 2 years ago. Her regular pursuits were affected because of her ankle deformity. She was also facing difficulty in using her shoes and was unable to participate in many recreational activities.

On examination, she was of average built, her vitals were stable, and the systemic examination was unremarkable. There were visible swellings at both distal thighs, left proximal thigh, bilateral proximal tibia, and the left distal leg. The swellings were non-tender, hard in consistency, attached to the underlying bone, free from the surrounding soft tissue, and with healthy overlying skin. The child had a two $\mathrm{cm}$ shortening on the left leg with a valgus deformity over the left ankle. The joint line and other bony prominences were non-tender. The ankle dorsiflexion was restricted with a range of $0-10^{\circ}$. There was no ankle instability or any distal neurovascular deficit.

On radiological evaluation, the distal fibular physis was proximal to the distal tibial physis, and the latter was triangular with a thinner lateral portion (Fig. 1). So, the patient had a grade three ankle valgus deformity according to the Malhotra et al. classification [3]. The tibiotalar angle, measured between the longitudinal axis of the tibia and the plane of the superior surface

Correspondence to: Dr. Gunjar Jain, Department of Orthopaedics, All India Institute of Medical Sciences, Ansari Nagar, New Delhi, India. E-mail: drgunjarjain@gmail.com

(C) 2021 Creative Commons Attribution-NonCommercial 4.0 International License (CC BY-NC-ND 4.0). 
of the talus on a standing anteroposterior radiograph was $69^{\circ}$. This angle corresponds to $21^{\circ}$ of tibiotalar tilt, and further implies the severity of the ankle valgus deformity.

We planned to perform a temporary hemiepiphysiodesis of the distal medial tibial epiphysis using a TBP. As the osteochondral lesions were asymptomatic, we didn't excise them. Under general anesthesia, we made a $3 \mathrm{~cm}$ long longitudinal skin incision centering over the distal medial tibial physis. An extraperiosteal eight-plate was then transfixed, with two non-locking cannulated cancellous screws on both the sides of the physis under fluoroscopic guidance (Fig. 2). In the postoperative period, the patient was allowed to bear full weight without any cast or brace support.

At the latest visit, after 18 months of the surgery, the patient had experienced significant clinical improvement. She now can walk for a longer distance without experiencing any pain and also participates in minor recreational activities. The correction in deformity was appreciable clinically (Fig. 3). The dorsiflexion

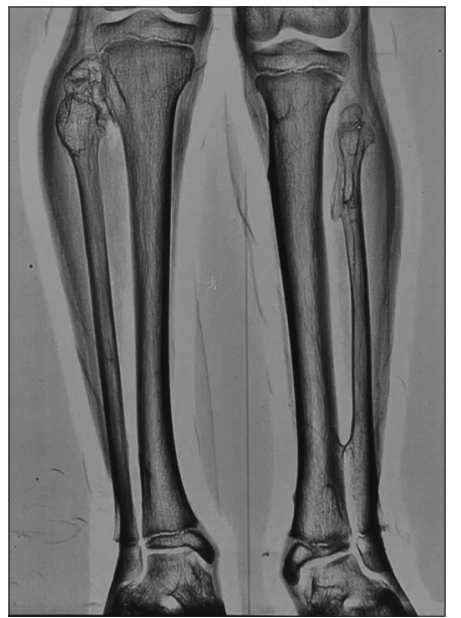

Figure 1: Preoperative anteroposterior standing radiograph of both legs: Note on left side exostoses is involving both proximal and distal tibiofibular joint with proximal tibiofibular diastasis, the distal fibular physis is proximal to the tibial physis, and there is a valgus deformity of the ankle with tapering of the distal tibial epiphysis on the lateral aspect

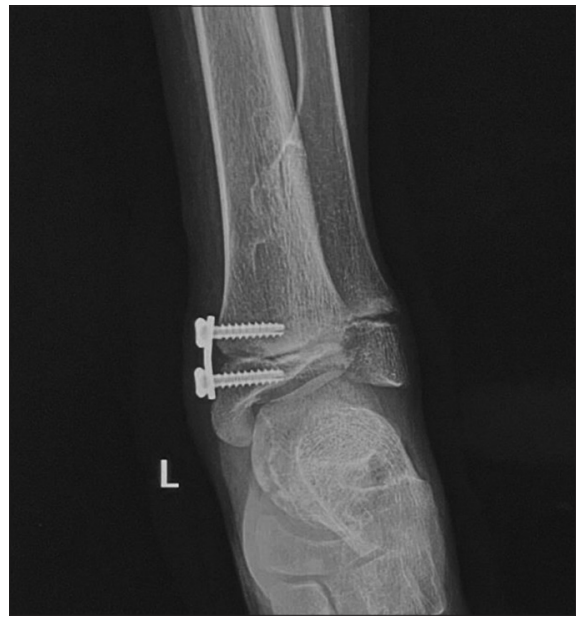

Figure 2: Immediate postoperative radiograph of left ankle showing tension band plate fixed across the medial distal tibial epiphysis. Note that the screws are applied parallel to each other of the left ankle was also improved to a range of $0-15^{\circ}$. There were no hardware-related or any other complications. In the latest weight-bearing radiographs, the tibiotalar angle was $80^{\circ}$ (Fig. 4). Thus, we have achieved a correction of $11^{\circ}$ at a rate of $0.61^{\circ} /$ month. Since the distal tibial physis is still open, the patient can achieve more correction. However, the Malhotra et al. grade did not improve. The fibular shortening slightly deteriorated with a relative fibula length of 0.91 , which was 0.92 preoperatively.

\section{DISCUSSION}

Temporary hemiepiphysiodesis is the most popular method for ankle valgus correction in the pediatric population. It is a minimally invasive procedure and does not require any weight-bearing restriction or immobilization. One disadvantage of temporary epiphysiodesis is the need for multiple follow-ups. Our case report highlights the importance of regular follow-up and a detailed evaluation at each visit to decide the ideal time for implant removal initially and look for any rebound deformity

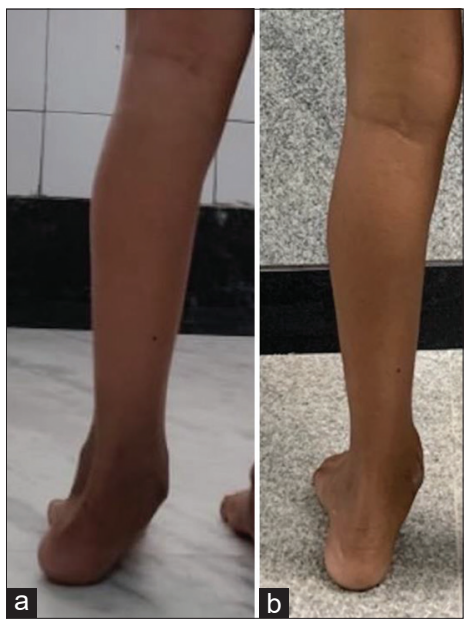

Figure 3: Standing clinical image (Posterior view) preoperatively (a) and at eighteen months of the surgery (b)

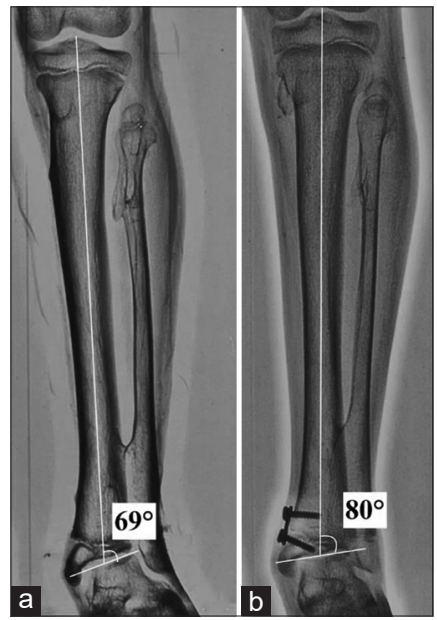

Figure 4: Anteroposterior standing radiograph of both legs preoperatively (a) and at one year and six months of the surgery (b). Note in the current radiograph that the distal fibular physis is still proximal to the tibial physis on left side, proximal tibiofibular diastasis is reduced, the screws have become divergent 
later. Overcorrected deformity, resulting from late follow-up and loss to follow-up, occurs in up to $39 \%$ of the cases [4]. A method to tentatively estimate the ROC preoperatively and the time needed to achieve normal alignment would allow for a more liberal follow-up protocol.

A timely growth modulation would help achieve the desired correction before the fusion of the distal tibial physis. The age of our patient was 9 years at the time of initial surgery. Therefore, sufficient growth was present to attain the desired correction. Gupta et al. had suggested that to achieve a $20^{\circ}$ correction, a minimum of 2 years of growth must be remaining [5]. On the contrary, hemiepiphysiodesis in a younger patient can cause a rebound deformity following the removal of the device. The latter can occur in up to $50 \%$ of patients and require a repeat hemiepiphysiodesis [6]. Therefore, the preoperative calculation of the ROC would also help prevent repeated surgeries by estimating an ideal age for surgery.

The primary pathology causing the deformity may also influence the time required for correction. However, there is no consensus among different authors regarding this. In the study of Driscoll et al., the mean rate of tibiotalar angle correction in HME ankles was $0.37 \%$ month, while that in non-HME ankles was $0.51 \%$ month [7]. The risk of recurrence was also higher in HME ankles than non-HME ankles [6]. However, Rupprecht et al. had opined that the primary etiology causing the ankle valgus doesn't influence the ROC after growth modulation [8]. In the current case, we have achieved a correction rate of $0.61 \%$ month in an HME ankle. This case shows that an adequate ROC can be achieved even in HME patients.

We had initiated the treatment in our case at 9 years of age and obtained a modest alignment after 18 months of surgery. In our case, the deformity was $21^{\circ}$, which was severe, and we have achieved a moderate ROC of $0.61^{\circ}$ /month. Van Oosterbos et al. had found that younger patients achieve greater correction compared to older children [9]. However, Rupprecht et al. had attained a higher mean ROC of $0.63 \%$ month even in relatively older patients with a mean age of 11.6 years [6]. On comparing the results of different authors, it appears that the ROC is not related to the age of the patient (Fig. 5) [10,11]. However, the mean ROC recorded by authors with more severe initial deformity was moderately higher compared to authors with relatively milder deformity (Fig. 6).

In the present study, we got the correction rate of $0.61 \%$ month by TBP. However, most other investigators using TBP, except for Stevens et al. [12], have achieved a lower mean ROC compared to authors using a transphyseal screw (Fig. 7). Despite this apparent slow correction, many authors prefer TBP over MMS as the latter violates the physis and is more difficult to remove due to bony overgrowth [12]. Furthermore, TBP more conveniently gets implanted in the mid-coronal plane compared to MMS or HS, thus less commonly leads to undesired deformities in other planes $[13,14]$. Due to its non-locking construct and pivoting of the screws, the TBP, compared to HS, causes a peripheral physeal tethering and allows limited growth [14]. Because of this property, it results in less hardware excursion, screw breakage,
Comparision of mean rate of correction and age at surgery of different studies

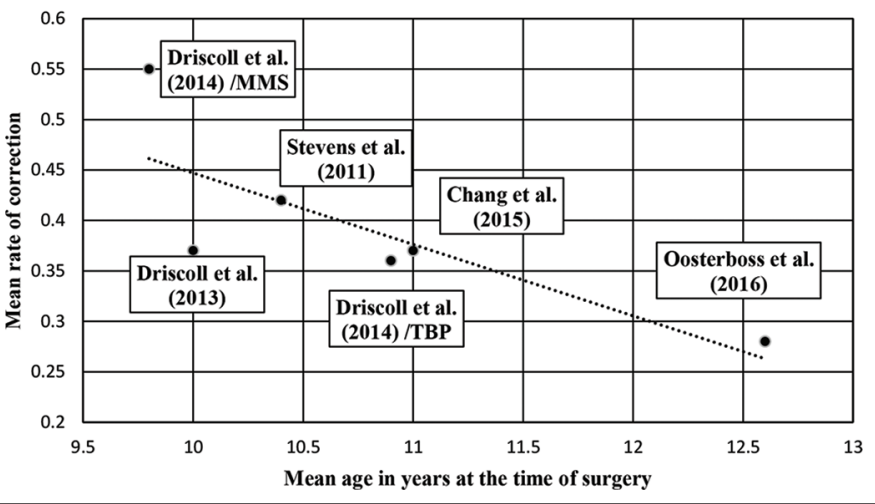

Figure 5: Comparison of the mean rate of correction vs mean age in studies of Driscoll et al. (2013) [6], Van Oosterbos et al. [8], Rupprecht et al. [9], Driscoll et al. (2014) [6], Chang et al. [10], Stevens et al. [11], and Tompkins et al. [12], Driscoll et al. had mentioned the data of patients treated with medial malleolar screw and tension band plate separately thus we have included them individually [6]

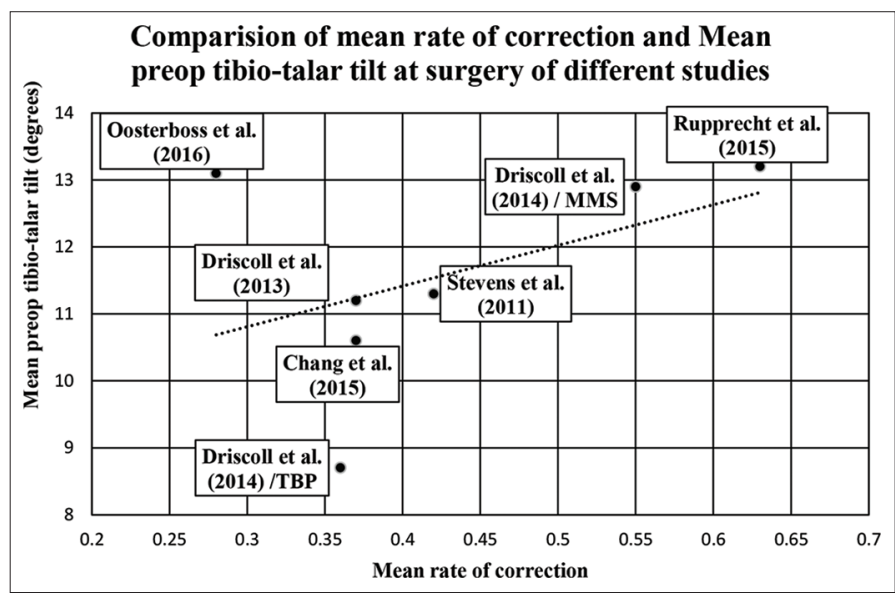

Figure 6: Comparison of the mean rate of correction vs tibiotalar tilt angle in studies of Driscoll et al. (2013) [7], Van Oosterbos et al. [9], Rupprecht et al. [10], Driscoll et al. (2014) [11], Chang et al. [12], and Stevens et al. [13]. Driscoll et al. had mentioned the data of patients treated with medial malleolar screw (MMS) and tension band plate (TBP) separately thus we have included them individually [11]

and physeal bar formation [14]. However, one demerit of TBP is hardware prominence. In the current case, we had advised the patient to avoid wearing any anklet or any shoe, causing direct pressure over the implant, but despite the subcutaneous position of the implant, she didn't experience any hardware-related discomfort.

In the present case, there was a significant fibular shortening, which did not improve with growth modulation. Some of the earlier surgeons have advised a concomitant fibula lengthening procedure in grade three shortening [15]. However, most of the recent reports regarding ankle valgus in HME patients did not mention the need for fibula lengthening even in severe deformities.

A very young child with HME and an ankle valgus deformity is usually managed only by excision of distal exostosis. It does not correct the alignment but reduces the pain and discomfort 


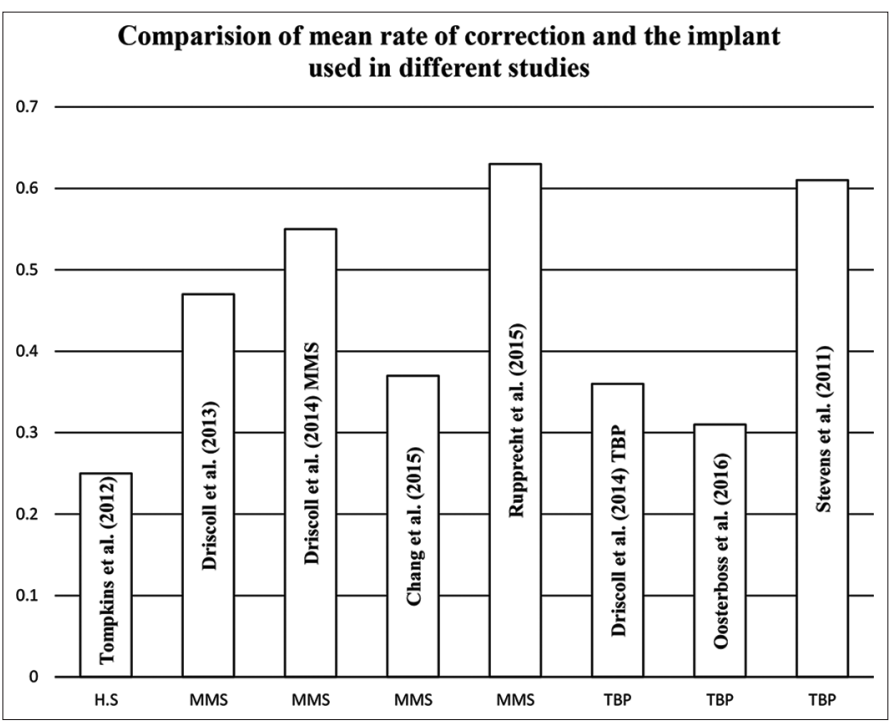

Figure 7: Comparison of the mean rate of correction with different implant devices in studies of Driscoll et al. (2013) [7], Van Oosterbos et al. [9], Rupprecht et al. [10], Driscoll et al. (2014) [11], Chang et al. [12], Stevens et al. [13], and Tompkins et al. [14], Driscoll et al. had mentioned the data of patients treated with medial malleolar screw (MMS) and tension band plate (TBP) separately thus we have included them individually [11]. H.S: Hemiepiphyseal Stapling.

associated with the deformity [15]. Sometimes an exostosis can directly cause increasing valgus deformity by a tethering effect [2]. In this situation, excision of the lesion can prevent progressive deformity and thus is advisable. Otherwise, a concomitant excision of the exostosis, along with hemiepiphysiodesis, is required if the lesion is painful or causing any compression symptoms.

\section{CONCLUSION}

From the current case, we can conclude that a necessary ROC of ankle valgus deformity can be obtained with medial malleolar temporary hemiepiphysiodesis using a TBP. The time required to achieve a normal alignment may depend on many factors including the age of the patient, the initial tibiotalar tilt, the implant used for surgery, and the pathological cause of the deformity.

\section{ACKNOWLEDGMENT}

Nil.

\section{REFERENCES}

1. Noonan KJ, Feinberg JR, Levenda A, Snead J, Wurtz LD. Natural history of multiple hereditary osteochondromatosis of the lower extremity and ankle. J Pediatr Orthop 2002;22:120-4.

2. Ahn YS, Woo SH, Kang SJ, Jung ST. Coronal malalignment of lower legs depending on the locations of the exostoses in patients with multiple hereditary exostoses. BMC Musculoskelet Disord 2019;20:564.

3. Malhotra D, Puri R, Owen R. Valgus deformity of the ankle in children with spina bifida aperta. J Bone Joint Surg Br 1984;66:381-5.

4. Lawing C, Margalit A, Ukwuani G, Sponseller PD. Predicting late follow-up and understanding its consequences in growth modulation for pediatric lower limb deformities. J Pediatr Orthop 2019;39:295-301.

5. Gupta P, Gupta V, Patil B, Verma V. Angular deformities of lower limb in children: Correction for whom, when and how? J Clin Orthop Trauma 2020;11:196-201.

6. Rupprecht M, Spiro AS, Schlickewei C, Breyer S, Ridderbusch K, Stücker R. Rebound of ankle valgus deformity in patients with hereditary multiple exostosis. J Pediatr Orthop 2015;35:94-9.

7. Driscoll M, Linton J, Sullivan E, Scott A. Correction and recurrence of ankle valgus in skeletally immature patients with multiple hereditary exostoses. Foot Ankle Int 2013;34:1267-73.

8. Rupprecht M, Spiro AS, Breyer S, Vettorazzi E, Ridderbusch K, Stücker R. Growth modulation with a medial malleolar screw for ankle valgus deformity. 79 children with 125 affected ankles followed until correction or physeal closure. Acta Orthop 2015;86:611-5.

9. Van Oosterbos M, van der Zwan AL, van der Woude HJ, Ham SJ. Correction of ankle valgus by hemiepiphysiodesis using the tension band principle in patients with multiple hereditary exostosis. J Child Orthop 2016;10:267-73.

10. Driscoll MD, Linton J, Sullivan E, Scott A. Medial malleolar screw versus tension-band plate hemiepiphysiodesis for ankle valgus in the skeletally immature. J Pediatr Orthop 2014;34:441-6.

11. Chang FM, Ma J, Pan Z, Hoversten L, Novais EN. Rate of correction and recurrence of ankle valgus in children using a transphyseal medial malleolar screw: J Pediatr Orthop 2015;35:589-92

12. Stevens PM, Kennedy JM, Hung M. Guided growth for ankle valgus. J Pediatr Orthop 2011;31:878-83.

13. Tompkins M, Eberson C, Ehrlich M. Hemiepiphyseal stapling for ankle valgus in multiple hereditary exostoses. Am J Orthop (Belle Mead NJ) 2012;41:E23-6.

14. Jelinek EM, Bittersohl B, Martiny F, Scharfstädt A, Krauspe R, Westhoff B. The 8-plate versus physeal stapling for temporary hemiepiphyseodesis correcting genu valgum and genu varum: A retrospective analysis of thirty-five patients. Int Orthop 2012;36:599-605.

15. Snearly WN, Peterson HA. Management of ankle deformities in multiple hereditary osteochondromata. J Pediatr Orthop 1989;9:427-32.

Funding: None; Conflict of interest: None of the authors have any conflicts of interest.

How to cite this article: Datt R, Jain G, Nag HL, Shekhar S. Management of ankle valgus in a skeletally immature patient by tension band plate hemiepiphysiodesis: A case report. Indian J Case Reports. 2021;7(10):461-464. 